\title{
A setup for ultrasound based assessment of the aortic root geometry
}

\author{
Hagenah $\mathrm{J}^{1}$, Scharfschwerdt $\mathrm{M}^{2}$, Stender $\mathrm{B}^{1}, \mathrm{Ott}^{1}$, Friedl $\mathrm{R}^{3}$, Sievers $\mathrm{HH}^{2}$, Schlaefer $\mathrm{A}^{1}$ \\ ${ }^{1}$ Medical Robotics, Institute for Robotics and Cognitive Systems, Universität zu Lübeck, Germany \\ ${ }^{2}$ Department of Cardiac Surgery, University Hospital Schleswig-Holstein, Lübeck, Germany \\ ${ }^{3}$ Klinik für Herz-, Thorax- und Gefäßchirurgie, Klinikum Karlsburg, Karlsburg, Germany \\ schlaefer@rob.uni-luebeck.de
}

\begin{abstract}
Planning aortic valve reconstruction requires a model of the aortic root geometry. One image modality available in the clinic is transesophageal ultrasound. We present a setup to obtain ultrasound images of porcine valves in different states, i.e., mimicking the dilatation and reconstruction of the aortic root. We studied whether key geometry parameters can be obtained from the ultrasound images. Our preliminary results indicate that the location of the commissures, the diameter of the annulus and the coaptation height can be estimated from the images and the setup can help validate the fitting of the prosthesis.
\end{abstract}

Keywords: aortic valve reconstruction, ultrasound, treatment planning

\section{Introduction}

Aortic valve reconstruction presents an alternative to valve replacement, particularly for younger patients. The main idea is to remodel the aortic root such that the function of the valve is restored, i.e., the cusps show sufficient coaptation and regurgitation is eliminated. Two methods for reconstruction have been proposed, the David and Yacoub procedures [1]. For both procedures, a prosthesis is used to shape the aortic root.

However, deciding the exact shape of the prosthesis and the geometry of the aortic root is an intricate task during surgery. The actual geometry can only be studied when the heart is pressurized, which is not the case during surgery. We have previously proposed a pre-treatment planning method based on soft tissue modelling and interactive variation of key parameters, e.g., the position of the commissures and the diameter of the annulus [2,3].

One challenge is the evaluation of the actual valve geometry for different situations while the valve remains under pressure [4]. We report the design of a setup to obtain parameters for the valve geometry from ultrasound images and present first results showing how it can be used to evaluate the coaptation for actual valves.

\section{Methods}

We have developed a setup to obtain ultrasound images of the aortic root where the pressure can be adjusted. The aorta is attached to a water filled tube as shown in Figure 1. A box filled with water and lined with foam holds the valve and an ultrasound probe. To mimic a realistic scenario we use a transesophageal echocardiography (TEE) probe connected to a GE vivid 7 ultrasound sys- tem. A pump is used to maintain the water level and hence the pressure on the valve.

Two dimensional ultrasound images are acquired sequentially by rotating the imaging plane. The images are converted into a Cartesian coordinate frame to represent a volumetric image of the aortic root.

To study whether the setup can be used to acquire information on the geometry of the root, a porcine valve was prepared and attached to the system. After image acquisition, the valve was manually modified to mimic a dilatation and another ultrasound volume was acquired. Finally, a prosthesis was used to emulate repairing the valve, followed by the acquisition of a further ultrasound volume. The three states are illustrated in Figure 2.

For each of the ultrasound volumes, natural landmarks like the commissures and the contact area between the valves were identified. The positions of the three commissures were used to define a similar coordinate frame for all three volumes. After transforming the images accordingly, the coaptation height was estimated.

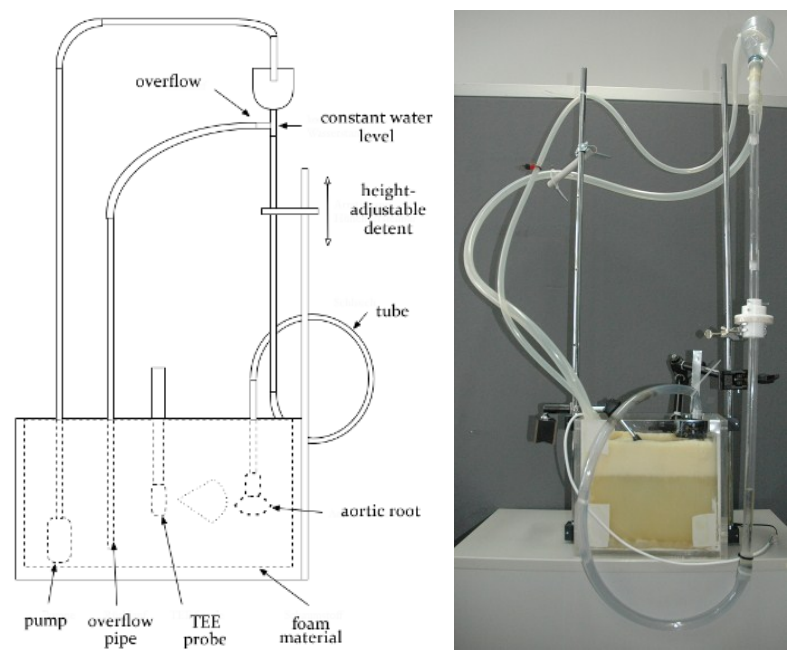

Figure 1: The setup for ultrasound acquisition with adjustable height, water circulation, and TEE probe
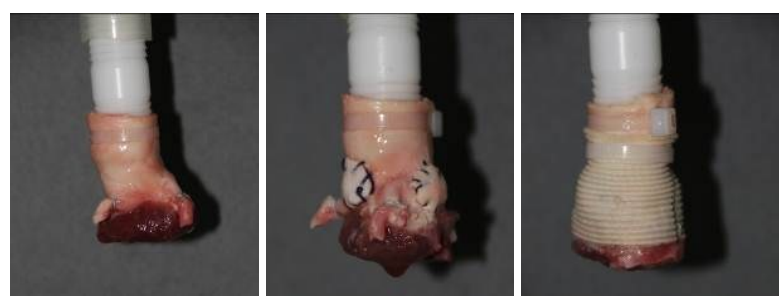

Figure 2: The three different states during the experiments: the original (left), dilated (center), and reconstructed root (right) 


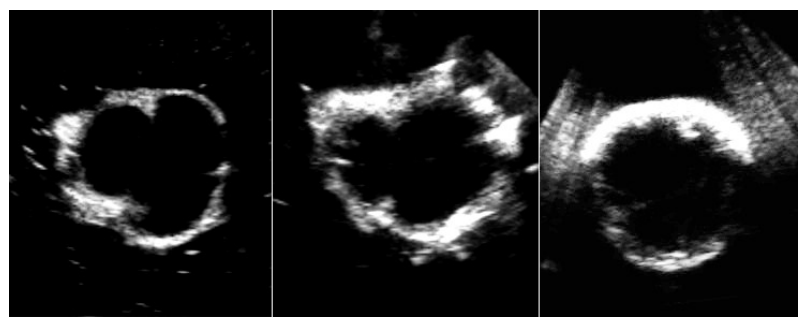

(a)

(b)

(c)

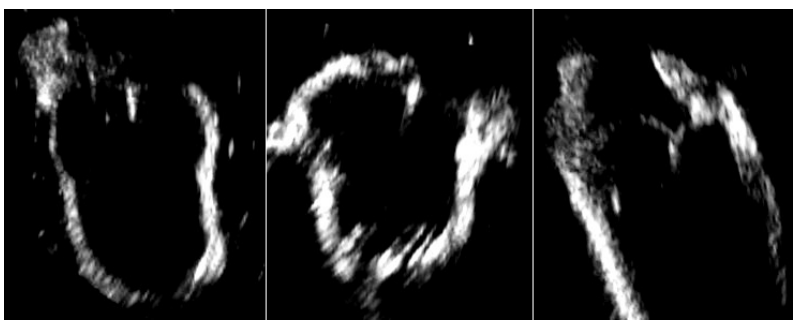

(d)

(e)

(f)

Figure 3: a cut above the commissures and a cut through the coaptation for original $(\mathrm{a}, \mathrm{d})$, dilated $(\mathrm{b}, \mathrm{e})$, and reconstructed $(\mathrm{c}, \mathrm{f})$ root, respectively

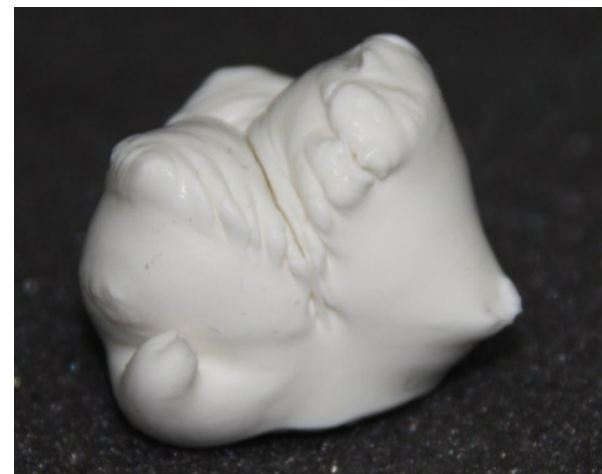

Figure 4: A silicon model of the aortic root

\section{Results}

The setup was used to obtain ultrasound volumes for a series of artificially dilated porcine aortic roots. Figure 3 shows a cut above the commissures and another cut through the coaptation area of the cusps for the three different states, respectively.

The coaptation height measured in the aligned images was $5.1 \mathrm{~mm}, 4.2 \mathrm{~mm}$, and $5.3 \mathrm{~mm}$ for the original, dilated, and reconstructed shape, respectively. The height of the commissures was approximately $8.2 \mathrm{~mm}, 7.4 \mathrm{~mm}$, and $8.2 \mathrm{~mm}$ for the three states.

\section{Discussion}

Recent work on simulating the aortic root function suggests that a careful planning of its geometry is critical for successful reconstruction $[2,3,5]$. However, so far the geometry was obtained from CT data [2,3] or an artificial model was built [5].

Our experiments have two interesting results. First, they illustrate that TEE may be used to obtained parameters for the geometric model of the aortic root. This would allow patient specific modelling based on a clinically available image modality. Second, the proposed setup presents an approach to evaluate and validate numerical simulations. The quantitative results obtained for the different states are realistic. To further assess the accuracy of the ultrasound based measurements, we are currently making silicon models of the root while in place in our setup, compare Figure 4.

\section{Bibliography}

[1] Yacoub M., Fagan A., Tassano P., Radley-Smith R. Result of valve conserving operations for aortic regurgitation. Circulation 1983; 68(suppl 3):321

[2] Hadjar H, Friedl R, Sievers HH, Hunold P, Stender B, Schlaefer A. Surgical planning tool for the simulation of the aortic valve-sparing surgery. Computer Assisted Radiology and Surgery (CARS 2011), 2011

[3] Hadjar H, Friedl R, Sievers HH, Hunold P, Stender B, Schlaefer A. Patient-specific finite-element simulation of aortic valve-sparing surgery. Computer Assisted Radiology and Surgery (CARS 2012), 2012

[4] Scharfschwerdt M, Sievers HH, Hussein A, Kraatz EG, Misfeld M. Impact of progressive sinotubular junction dilatation on valve competence of the $3 \mathrm{~F}$ Aortic and Sorin Solo stentless bioprosthetic heart valves. Eur J Cardiothorac Surg 2010, 37: 3. 631-634

[5] Marom G, Haj-Ali R, Rosenfeld M, Schäfers HJ, Raanani E. Aortic root numeric model: annulus diameter prediction of effective height and coaptation in postaortic valve repair. J Thorac Cardiovasc Surg. 2013 Feb;145(2):406-411 